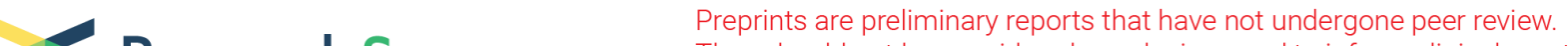 Research Square They should not be considered conclusive, used to inform clinical practice, or referenced by the media as validated information.
}

\section{Disrupted fronto-temporal function in panic disorder: a resting-state connectome study}

\section{Yun Wu ( $\square$ YunW1010@163.com )}

Nanjing Brain Hospital https://orcid.org/0000-0002-0080-408X

\section{Yuan Zhong}

Nanjing Normal University

\section{Gang Zheng}

Department of Medical Imaging, Medical Imaging Center, JinLing Hospital

\section{Ya Liu}

Medical Imaging Center, Jinling Hospital

\section{Manlong Pang \\ Nanjing Brain Hospital \\ Huazhen Xu \\ Nanjing Brain Hospital \\ Huachen Ding \\ Nanjing Brain Hospital}

\section{Chun Wang}

Nanjing Brain Hospital

Ning Zhang

Nanjing Brain Hospital

\section{Research Article}

Keywords: magnetic resonance imaging, graph theory, network-based statistic, temporal lobe

Posted Date: June 23rd, 2021

DOl: https://doi.org/10.21203/rs.3.rs-240681/v1

License: (c) (i) This work is licensed under a Creative Commons Attribution 4.0 International License. Read Full License

Version of Record: A version of this preprint was published at Brain Imaging and Behavior on October 19th, 2021. See the published version at https://doi.org/10.1007/s11682-021-00563-z. 


\section{Abstract}

Recent neuroimaging studies have identified altered activations and connectivity among many brain regions as potential biomarkers for panic disorder. However, little was known about how topological properties would change in panic disorder. Therefore, a graph-theoretical approach was applied in this study to construct functional networks of patients and healthy controls to discover topological changes in panic disorder. 31 patients and 33 matched healthy controls underwent resting-state functional magnetic resonance imaging. Brain network of each participant was structured using the Anatomical Automatic Labeling template as nodes and connectivity matrixes as edges. Then, topological organizations of networks were calculated. Network-based statistic analysis was conducted and global and nodal properties were compared between patients and controls. Patients with panic disorder showed small-world attribute, which was lower than that in controls. Patients revealed decreased nodal efficiency in superior and middle frontal gyrus, right superior temporal gyrus and left middle temporal gyrus. Decreased functional connectivity was found in panic disorder between right middle temporal gyrus and extensive temporal regions. Results indicated decreased function of global and regional information transmission in panic disorder, highlighted the disrupted "top-down" processing in fronto-temporal regions and emphasized the role of temporal regions in the pathology of panic disorder.

\section{Introduction}

Patients with panic disorder (PD) suffer from core symptom of recurring panic attacks which is often manifested as palpitations, dizziness, accelerated heart rate, trembling, sweating and fear of going crazy or even dying (Sobanski \& Wagner, 2017). Besides panic attacks, PD patients also experience related cognitive and behavioral changes resulting in anticipatory anxiety and avoidance behaviors. Epidemiological study showed PD's lifetime prevalence is 4.7\% (Kessler et al., 2006).

\subsection{Fear network hypothesis in PD}

The fear network hypothesis was the first modal raised by Gorman (Gorman \& M., 2000) in the neuropathology of $\mathrm{PD}$, which pointed that overactivation of amygdala and disrupted inhibitive function of prefrontal cortex (PFC) were the main cause of panic attacks. This modal has been supported by many studies reporting abnormal top-down processing (Kent et al., 2005; Lueken, Straube, Reinhardt, Maslowski, \& Kircher, 2014; Nash et al., 2008). Except PFC and limbic regions, recently sensory regions including temporal and occipital regions were reported to be changed in PD. Lai proposed an advanced modal of fear network, adding temporal, parietal and occipital regions in the modal (C. H. Lai, 2019), which emphasized disrupted sensory information transmission from sensory regions to frontal regions. In line with Lai's hypothesis, our previous meta-analysis of voxel-based morphometry (VBM) studies also identified decreased gray matter volume (GMV) in middle temporal gyrus (MTG) and superior temporal gyrus (STG) in PD (Wu et al., 2018). Changes in extensive temporal regions have been reported repeatedly but with different subregions such as STG, MTG and inferior temporal gyrus (ITG), and inconsistent 
change directions (Lee et al., 2006; Spoormaker et al., 2011). The role of different temporal regions in the pathology of PD remains unclear.

\subsection{Introduction of functional connectome analysis}

For its stability and convenience of implementation, resting-state functional magnetic resonance imaging ( $\mathrm{FMRI}$ ) is often used to identify abnormal activations of brain regions and functional connectivity (FC) in psychiatry researches. Recently, the emerging method of connectome analysis based on graph theory has been applied in psychiatric studies, such as schizophrenia (Liu et al., 2008), MDD (Zhang et al., 2011) and anxiety disorders like social anxiety disorder (Zhu et al., 2017). This method provides a way of understanding mental illnesses from the angle of information transmission between brain regions. It has been widely proved that human brain is characterized by small-worldness under multiple task conditions (Eguiluz, Chialvo, Cecchi, Baliki, \& Apkarian, 2005), as well as in resting-state conditions (van den Heuvel, Stam, Boersma, \& Hulshoff Pol, 2008), which implies both high global efficiency of information transmission across the whole brain and high local efficiency within each local functional network. Patients with psychiatric disorders were found to have decreased small-worldness (Liu et al., 2008; L. Wang et al., 2009).

\subsection{Objectives of the study}

Although there have been many psychiatric studies focusing on connectome changes, we found only one study conducting graph-based network analysis on PD (C. H. Lai \& Wu, 2016). Lai's study reported connectome changes of $\mathrm{PD}$, identifying extensive decreases of $\mathrm{FC}$ among regions, such as left parahippocampal gyrus and bilateral precentral gyrus, which emphasized the importance of limbic regions and the connection between sensory and motor regions. However, topological property changes were not mentioned in the study.

Therefore, the present study aimed at exploring the pathology of PD from a new perspective of information transmission in a more comprehensive way, including changes of FC and topological properties in PD.

\section{Materials And Methods}

\subsection{Participants}

The study aimed to enroll 40 patients with PD and 40 age- and gender-matched healthy controls (HCs). PD patients were recruited from Brain Hospital of Nanjing Medical University, and healthy participants were recruited from the community from September 2014 to January 2019. Patients were assessed for study eligibility as follows. The inclusion criteria for PD patients included: (1) PD diagnoses were made according to DSM-V criteria and the Chinese version of the Mini-International Neuropsychiatric Interview criteria; (2) Age between 18 and 55 years old; (3) Right-handed. Patients were excluded if they have the following conditions: (1) Neurological or other psychiatric illnesses; (2) Severe somatic diseases; (3) 
Contraindicated factors for MRI, such as claustrophobia, discomforts while undergoing MR examination, a pacemaker in the body, pregnant or nursing.

This project was approved by the Ethics Committee of the Nanjing Brain Hospital, affiliate of Nanjing Medical University. After complete explanation of the procedures to participants, written informed consent was obtained.

\subsection{Clinical assessments}

Clinical symptoms were measured through 3 scales. Panic Disorder Severity Scale (PDSS) was used by several trained psychiatrists to assess the severity of panic symptoms; Hamilton Anxiety Scale (HAMA) was used to assess the anxiety level of patients; The Chinese revision of the Anxiety Sensitivity Index (ASI-R) was also used to evaluate the anxiety sensitivity of patients.

\subsection{Resting-state fMRI acquisition}

All imaging data were obtained with a 3.0 T Siemens version scanner in the afternoon between 14:0016:00 as soon as the participants were induced in the study. Whole-brain fMRI was acquired using an 8min echo-planar imaging sequence (repetition-time/echo-time $=2000 / 30 \mathrm{~ms}$, flip angle $=90^{\circ}$ ). The slice thickness was $4 \mathrm{~mm}$ and the matrix size was $64 \times 64$, resulting in voxels of $3.75 \times 3.75 \times 4.0 \mathrm{~mm}^{3}$. Participants were instructed to close their eyes and rest, to stay awake and not to sleep. High-resolution T1-weighted structural MRI was obtained for subsequent process of BOLD data co-registration using three-dimensional spoiled gradient-echo sequence (repetition-time/echo-time $=1900 / 2.48 \mathrm{~ms}$, voxels $1 \times$ $\left.1 \times 1 \mathrm{~mm}^{3}\right)$.

\subsection{Data preprocessing}

Functional data were preprocessed using the Data Processing Assistant for Resting-State fMRI (DPARSF_V4.0) in DPABI (http://rfmri.org/dpabi) (Yan, Wang, Zuo, \& Zang, 2016). This toolbox is based on Statistical Parametric Mapping (SPM8, http://www.fil.ion.ucl.ac.uk/spm) and the Matlab platform (http://www.brain-connectivity-toolbox.net). DPARSF is developed for more convenient preprocessing steps and widely used in resting-state fMRI studies (Chao-Gan \& Yu-Feng, 2010). To attain magnetization equilibrium, first 10 time points were discarded. For each participant, images were slice-timing corrected using sinc interpolation and then realigned. After head motion correction, linear regression was performed to remove Friston's twenty-four head motion parameters and confounding signals of white matter, cerebrospinal fluid and global signals. All the images were normalized by using each participant's T1 image unified segmentation and spatially smoothed with a 6-mm full-width half maximum Gaussian kernel. Finally, temporal band-pass filtering was performed using a cut-off frequency of $0.01-0.1 \mathrm{~Hz}$. Participants whose head motion exceeded $2.5 \mathrm{~mm}$ or 2.5 degrees were excluded.

\subsection{Network construction}

According to graph theory, a network is composed of nodes and edges between nodes. The atlas of Automated Anatomical Labeling (AAL) was used to represent 90 nodes of the network since it has been 
widely used in many neuroimaging studies (Tzourio-Mazoyer et al., 2002). The network edges were defined using resting-state $\mathrm{FC}$, which was calculated using pairwise Pearson correlations between any two regions' mean time series. Then, FC values were normalized to $z$-scores by Fisher's transformation. From this, a $90 \times 90$ correlation matrix, or a binarized (based on a range of sparsity), undirected graph was derived for each subject.

\subsection{Network-based statistic analysis}

A non-parametric approach called network-based statistic (NBS) (Zalesky, Fornito, \& Bullmore, 2010) was used to evaluate connectivity disruption by computing multiple test statistics while controlling the familywise error rate. NBS analysis was conducted using GRETNA toolkit (J. Wang et al., 2015) to find differences between PD and HC groups with age, gender, education information as covariates.

The NBS approach included following steps. First, a set of supra-threshold links were identified using tstatistic with an uncorrected threshold of $p<0.001$. Then a breadth first search (Ahuja, Magnanti, \& Orlin, 1994) was used to determine connected components in which any two voxels were connected to each other through a path comprising supra-threshold links. The number of links in each connected component (component size $\mathrm{N}$ ) was also stored at this step. Permutation testing was conducted with a $p$ value controlled for FWE ascribed to each connected component on the number of links it comprised. $A$ total of 10000 random permutations were generated independently, during which the group a participant belonged to (PD or $\mathrm{HC}$ ) was randomly exchanged. The permutation process yielded a null distribution for the size of the largest connected component so that a corrected $p$-value could be determined by calculating the proportion of permutations for which the largest connected component size was larger than N. The result of altered components in PD was visualized by BrainNet Viewer (Xia, Wang, \& He, 2013).

\subsection{Graph-theory network analysis}

Graph-theory metrics were calculated using GRETNA. Both global measures and local measures were used to describe the network's topological characteristics.

Global measures included: characteristic path length $\left(L_{p}\right)$, clustering coefficient $\left(C_{p}\right)$, global efficiency $\left(E_{g}\right)$, small-worldness $(\sigma)$. $L_{p}$ is defined as the average of the shortest path lengths (the minimum number of edges from a particular node to another) between any pair of nodes in the network. $C_{p}$ describes the extent of local connectivity of a network and is defined as the average clustering coefficients (see below) of all nodes in the network. $E_{g}$ measures the ability of information transmission of a network, which is defined by the average inverse path length of the shortest paths connecting all nodes in a network (Gong et al., 2009). High global efficiency implies fast information transmission and close topological relationship of nodes throughout the network.

Small-worldness is a parameter integrating the efficiency of both local and global information transfer. A small-world network is defined by high $C_{p}$ and low $L_{p}$ (Watts \& Strogatz, 1998). Small-worldness was 
calculated as the ratio of the $\mathrm{C}_{\mathrm{p}}$ of actual brain network to that of a random network ( $\mathrm{Y}$ ) divided by the ratio of the $L_{p}$ of real brain network to that of a random network $(\lambda)$ (Bassett, Brown, Deshpande, Carlson, \& Grafton, 2011; Humphries \& Gurney, 2008).

Local measures included: nodal degree centrality, nodal betweenness centrality, nodal global efficiency

and nodal clustering coefficient $\left(\mathrm{E}_{\mathrm{loc}}\right)$. Nodal degree centrality is defined by the total number of edges of a particular node divided by maximum possible edges the node could have with other nodes. Higher nodal degree centrality means more importance of this particular node in the network. Betweenness centrality is calculated as ratio of the number of shortest paths between any two nodes traversing a particular node to the number of all the shortest paths between any two nodes. This parameter is often used to define hubs which may is crucial in the network and closely connected to other nodes. Nodal global efficiency reflects the efficiency of information transmission from one particular node to other nodes. Clustering coefficient is defined as the number of connections between neighbor nodes of a particular node divided by maximum possible number of such nodes. High clustering coefficient implies a more specialized and integrated topological organization and good defense ability of a network against attack.

All the graph-theory metrics were calculated at a range of sparsity from $5-40 \%$ of the strongest connections within connectivity matrix. For each metric, area-under-the-curve (AUC) was calculated across all values of sparsity in $1 \%$ intervals. These AUC values were used for statistical comparisons.

\subsection{Statistical analyses}

A general linear model was performed with each global / regional network metric as independent variables and age, gender, education information as covariates. For multiple comparison correction, an alpha level of 1/90 ( $p<0.01)$ was used to declare significance for regional properties (Lynall et al., 2010). All the comparison processes were conducted using GRETNA toolkit.

The relationship between topological metrics with significant group differences and clinical symptoms of PD was calculated using Spearman's correlation coefficient.

\section{Results}

3.1 Demographic data and clinical symptoms

A total of 31 patients with PD (17 males and 14 females) and 33 HCs (14 males and 19 females) were recruited in the study. At the time of MR scanning, 24 out of 31 patients received no medications or psychological treatments; three patients were taking escitalopram; two patients were taking lorazepam and escitalopram and one patient was taking fluvoxamine. After preprocessing, MRI data of 1 PD and 4 HCs were discarded due to high head motion.

No significant differences of age, gender and years of education were found between two groups (Table 1). 


\subsection{Altered functional connectivity in PD}

NBS analysis identified a single network showing significant decreased FC in PD patients compared to $\mathrm{HC}(p=0.048$, FWE corrected). A total of 5 nodes and 4 edges were involved (Figure 1A, Table 2). Nodes with altered FC were mainly in temporal lobe in right hemisphere. Right MTG were involved in all 4 altered edges, indicating the significance of MTG in PD. The correlation analysis found the FC between right MTG and right transverse temporal gyrus showed significant positive correlation with HAMA score (Figure 1B).

\subsection{Disrupted global properties in PD}

Global properties under different connection sparsity were depicted in Figure 2. PD and HC groups all had small-world properties $(\sigma>1)$. The comparison of the AUC of $Y$ and $\lambda$ yielded no significant difference between PD and HC ( $\mathrm{:} t=-1.716, p=0.092 ; \lambda: t=1.339, p=0.186)$. Compared with HC group, the AUC value of $\sigma$ was significantly lower in PD group $(t=-2.21, p=0.03)$ (Figure 3$)$. The decreased $\gamma$ and increased $\lambda$ indicated that both global and local information transfer efficiency had the tendency of decreasing in PD patients, which attributed to the significant decrease of small-worldness of PD patients. No significant correlations were found between the AUC value of $\sigma$ of PD group and the PDSS score $(r=-0.303, p=0.104)$, between the AUC of $\sigma$ and the ASI score $(r=-0.001, p=0.995)$ and between the AUC of $\sigma$ and the HAMA score $(r=-0.048, p=0.800)$.

\subsection{Disrupted regional properties in PD}

The comparison of regional properties between PD and HC showed PD patients had increased higher nodal degree centrality in right posterior cingulate gyrus and left superior occipital gyrus, decreased higher nodal degree centrality in right superior frontal gyrus (SFG) and left MTG; PD patients had increased nodal betweenness centrality in right rectus and left angular gyrus; Decreased nodal global efficiency were found in extent frontal and temporal regions of PD patients (Table 3). The comparison of nodal clustering coefficient yielded no significant group difference.

The brain-symptom correlation analysis showed that the AUC of nodal global efficiency of right SFG had significant negative correlation with ASI-R score (Figure 4A); the AUC of nodal betweenness centrality of left angular gyrus had significant positive correlation with HAMA score (Figure 4B).

\section{Discussion}


This study characterized resting-state network alterations in PD from two perspectives: both connectivity and topological property changes. To our knowledge, this is the first study to investigate network changes in PD using both NBS analysis and topological measures. Small-worldness attribute comparison revealed that PD patients showed lower small-worldness than HC, indicating the tendency of decreased global and local network efficiency in PD. The abnormalities may have correlation with loss of long-range communication among parts of the brain in PD (Latora \& Marchiori, 2001).

The comparison of nodal global efficiency revealed lower regional topological property in right SFG, right MFG, right STG and left MTG. Frontal lobe is an essential part in the neuropathology of PD since fear network hypothesis was raised in 2000 (Gorman \& M., 2000). The disruption of SFG has been widely reported in many studies (Feldker et al., 2016; Killgore et al., 2014; Wedekind et al., 2011). SFG and STG were found to be involved in the extinction phase in fear conditioning (Lueken et al., 2014; Sehlmeyer et al., 2009). In extinction circuitry, PFC inhibits the response of amygdala to regulate fear extinction (Quirk, Garcia, \& González-Lima, 2006; Sotres-Bayon, Cain, \& LeDoux, 2006). Our previous meta-analysis of VBM studies also found gray matter deficits in extent frontal regions and STG and co-atrophy between bilateral dorsomedial PFC, orbital frontal cortex (OFC) and STG (Wu et al., 2018), which provided evidence for decreased inhibitory function of PFC and STG from brain structural perspective. Decreased nodal global efficiency in frontal and temporal regions and negative correlation found between the AUC of nodal global efficiency of SFG and HAMA scores indicated disrupted long-range communication of "top-down" regulation in PD, which may lead to anxiety symptoms.

Decreased nodal degree centrality of right SFG and right MTG in PD conformed with the result of nodal network efficiency comparison mentioned above, which emphasized the crucial role of SFG and STG in "top-down" process of emotion regulation in PD. Nodal degree centrality comparison also found increased degree centrality in right PCC and left superior occipital gyrus in PD. Although PCC is not a typical part of fear network, PCC changes has been reported repeatedly in structural and functional MRI studies. Gray matter deficits (C.-H. Lai, Hsu, \& Wu, 2010) and higher fractional anisotropy (FA) (Han et al., 2008) were found in PCC in PD patients. Greater activation in PCC was found in PD while facing threatrelated stimuli (Maddock, Buonocore, Kile, \& Garrett, 2003). A recent fMRI study found increased activation in PCC in PD in breath-holding task and positive relationship between activation of PCC and self-reported fear (Mclntosh, Hoshi, \& Timpano, 2020). As a partial region involved in default mode network (DMN), PCC is correlated with self-referential and internally generated thoughts (Buckner, Andrews-Hanna, \& Schacter, 2008). Increased degree centrality in PCC may indicate overactivation of DMN posterior sub-network, which might cause excessive attention to internal and external treat-related stimuli in PD (Coutinho et al., 2016; C.-H. Lai \& Wu, 2014). Increased degree centrality in left superior occipital gyrus in PD proved "extent fear network" hypothesis (C. H. Lai, 2019). In Lai's advanced fear network model, occipital lobe might also be involved in fear identification and adaptation with thalamus, amygdala, frontal regions and other sensory regions (C. H. Lai, 2019; Leitman et al., 2008). Occipital lobe was associated with face recognition (Japee, Crocker, Carver, Pessoa, \& Ungerleider, 2009), visual spatial attention (Macaluso, Frith, \& Driver, 2000) and fearful stimuli processing (Bayle \& Taylor, 2010). Increased degree centrality in occipital gyrus indicated the abnormality of sensory information, especially fear 
signals processing and transmit to the fear network (van de Riet, Grezes, \& de Gelder, 2009), which might be related to the sensitivity of fearful stimuli and excessive anxiety in PD (Tauscher, Bagby, Javanmard, Christensen, \& Kapur, 2001).

Nodal betweenness centrality describes the significance of node in the network. Increased nodal betweenness centrality in left angular gyrus indicated excessive FC of angular gyrus with other regions in PD. Angular gyrus was also found to be involved in fear conditioning (Spoormaker et al., 2011). Decreased regional homogeneity was reported in angular gyrus in remitted PD patients after antidepressant treatment (C. H. Lai \& Wu, 2013). Our study also found positive relationship between betweenness centrality of angular gyrus and HAMA scores, which corresponded with previous studies showing angular gyrus abnormalities in patients with anxiety disorder (Eren, Tükel, Polat, Karaman, \& Unal, 2003; Liao et al., 2010; Qiu et al., 2011).

NBS analysis identified lower FC between MTG and other 4 regions, which were mainly in bilateral temporal lobe and left postcentral lobe. Decreased FC in extent temporal regions supported "extent fear network" hypothesis raised by Lai (C. H. Lai, 2019), which emphasized the role of temporal lobe in fear network. Corresponding with our previous meta-analysis of VBM studies of PD (Wu et al., 2018), MTG played a crucial role in the neuropathology of PD. Decreased GMV of MTG in PD was reported in structural MRI studies of PD (Massana et al., 2003; Sobanski et al., 2010). Different from structural studies, functional fMRI studies of PD showed inconsistent results. Higher activation in MTG was detected in PD patients during negative pictures viewing (Reinecke et al., 2015). As a crucial part of temporal lobe, MTG is involved in many functions: semantic processing (Carin, Elizabeth, \& Tilo, 2010; Mcdermott, Petersen, Watson, \& Ojemann, 2003), auditory stimuli processing (Mirz et al., 1999), facial expression processing (Sato, Toichi, Uono, \& Kochiyama, 2012) and reasoning (Goel, Gold, Kapur, \& Houle, 1998). FC abnormalities of MTG might indicate higher cognitive dysfunctions in PD except emotion regulation (Galderisi et al., 2008). Decreased FC between right MTG and right transverse temporal gyrus revealed disrupted information transfer in perceptive and cognitive function in PD. However, positive relationship between the $\mathrm{FC}$ of these two regions and anxiety symptoms may indicate different connectivity patterns between PD and HC.

There are several limitations in our study. First, some patients were taking medicines at the time of MRI scanning. Future studies could recruit drug-naive patients to reduce bias caused by sample heterogeneity; Second, the present study used AAL template, which divided the brain on the anatomical basis. The parcellation of the brain into meaningful regions remains an open issue (He \& Evans, 2010; Wang \& Jinhui, 2010; Wig, Schlaggar, \& Petersen, 2011). Random parcellation might also be needed. Third, the present study is cross-sectional, longitudinal studies are still needed to follow up the patients.

\section{Conclusion}

Our results suggested PD patients had decreased global and local network efficiency, which might be related to disrupted "top-down" processing in fronto-temporal regions in PD. Lower FC between MTG and 
other temporal regions might indicate the important role of temporal regions in the pathology of PD.

\section{Declarations}

\section{Funding}

This study was supported by National Natural Science Foundation of China [grant numbers 81971289 , 81871344, 81671667, 81471644]; Jiangsu Provincial key research and development program [grant number BE2019609]; Natural Science Foundation of Jiangsu Province [grant number BK20191369]; the Natural Science Foundation of the Higher Education Institutions of Jiangsu Province, China [grant number 18KJB190003].

\section{Conflicts of Interest}

All authors report no biomedical financial interests or potential conflicts of interest. This study has not been published previously and has not been reproduced from prior publications.

\section{Ethics approval}

This study was reviewed and approved by the Ethics Committee of the Nanjing Brain Hospital, affiliate of Nanjing Medical University.

Consent to participate

After complete explanation of the procedures to participants, written informed consent was obtained.

Acknowledgements

We would like to thank all the research assistants who supported the recruitment and scanning for this study.

Consent for publication

All the authors agreed to publish this study in this journal. 
The data that support the findings of this study are available from the corresponding author upon reasonable request.

Code availability

Not applicable

\section{Authors' contributions}

Author contributions included conception and study design (Chun Wang), acquisition of data (Manlong Pang, Huazhen Xu, Huachen Ding), statistical analysis (Yuan Zhong, Gang Zheng, Ya Liu), interpretation of results (Ning Zhang), drafting the manuscript work (Yuan Zhong) or revising it critically for important intellectual content (all authors) and approval of final version to be published and agreement to be accountable for the integrity and accuracy of all aspects of the work (all authors).

\section{Tables}

Table 1. Demographic and clinical information of PD and HC

\begin{tabular}{lllrc}
\hline & $\mathrm{PD}(\mathrm{n}=30)$ & $\mathrm{HC}(\mathrm{n}=29)$ & $T / c^{2}$ & $p$ \\
\hline Gender (Male/Female) & $16 / 14$ & $13 / 16$ & 0.43 & 0.51 \\
Age (years) & $34.93 \pm 8.01$ & $36.93 \pm 9.24$ & -0.89 & 0.38 \\
\hline Years of education & $13.33 \pm 3.54$ & $14.07 \pm 3.85$ & -0.76 & 0.45 \\
Illness Duration (years) & $2.08 \pm 3.04$ & & & \\
PDSS & $12.43 \pm 5.20$ & & & \\
ASI-R & $39.89 \pm 12.09$ & & & \\
HAMA & $17.77 \pm 8.19$ & & & \\
\hline
\end{tabular}

Data are presented as the range of minimum-maximum (mean \pm standard deviation).

PD, panic disorder; HC, healthy controls; PDSS represents the Panic Disorder Severity Scale; ASI-R represents the revision of the Anxiety Sensitivity Index.

The $p$ value of gender comparison was obtained using a chi-square test while other $p$ values were obtained using two-sample t-tests.

Table 2. Edges showing significant alterations between PD and HC

\begin{tabular}{lc}
\hline Edges & $t$ value \\
\hline Right middle temporal gyrus - Left postcentral gyrus & -3.484 \\
Right middle temporal gyrus - Left transverse temporal gyrus & -3.966 \\
\hline Right middle temporal gyrus - Right transverse temporal gyrus & -3.538 \\
\hline Right middle temporal gyrus - Right temporal pole: superior temporal gyrus & -3.681 \\
\hline
\end{tabular}


$T$ value $<0$ means $\mathrm{PD}<\mathrm{HC}$.

Table 3. Regional property changes in PD patients

\begin{tabular}{|c|c|c|}
\hline Regions & $t$ & $p$ \\
\hline \multicolumn{3}{|c|}{ Regions showing significant difference between PD and HC in nodal degree centrality } \\
\hline Right superior frontal gyrus & -3.116 & 0.003 \\
\hline Right posterior cingulate gyrus & 2.937 & 0.005 \\
\hline Left superior occipital gyrus & 3.276 & 0.002 \\
\hline Left middle temporal gyrus & -2.717 & 0.009 \\
\hline \multicolumn{3}{|c|}{ Regions showing significant difference between PD and HC in nodal betweenness centralit } \\
\hline Right rectus & 3.003 & 0.004 \\
\hline Left angular gyrus & 3.314 & 0.002 \\
\hline \multicolumn{3}{|c|}{ Regions showing significant difference between PD and HC in nodal global efficiency } \\
\hline Right superior frontal gyrus & -3.241 & 0.002 \\
\hline Right middle frontal gyrus & -2.695 & 0.009 \\
\hline Right superior temporal gyrus & -2.961 & 0.005 \\
\hline Right temporal pole: superior temporal gyrus & -2.764 & 0.008 \\
\hline Left middle temporal gyrus & -2.725 & 0.009 \\
\hline
\end{tabular}

\section{Regions showing significant difference between PD and HC in nodal clustering coefficient} none

$T$ value $>0$ means $\mathrm{PD}>\mathrm{HC} ; T$ value $<0$ means $\mathrm{PD}<\mathrm{HC}$.

\section{References}

Ahuja, Magnanti, T. L., \& Orlin, J. B., 1994. Network flows: Theory, algorithms and applications: Ravindra K. Ahuja, Thomas L. Magnanti and James B. Orlin (Prentice Hall, Englewood Cliffs, NJ, 1993) 846 pages. Discrete Applied Mathematics. 50. 99. http://doi.org/https://doi.org/10.1016/0166-218X(94)90171-6.

Bassett, D. S., Brown, J. A., Deshpande, V., Carlson, J. M., \& Grafton, S. T., 2011. Conserved and variable architecture of human white matter connectivity. Neuroimage. 54. 1262-1279.

http://doi.org/10.1016/j.neuroimage.2010.09.006.

Bayle, D. J., \& Taylor, M. J., 2010. Attention inhibition of early cortical activation to fearful faces. Brain Res. 1313. 113-123. http://doi.org/10.1016/j.brainres.2009.11.060.

Buckner, R. L., Andrews-Hanna, J. R., \& Schacter, D. L., 2008. The brain's default network: anatomy, function, and relevance to disease. Ann N Y Acad Sci. 1124. 1-38.

http://doi.org/10.1196/annals.1440.011.

Carin, W., Elizabeth, J., \& Tilo, K., 2010. Heterogeneity of the Left Temporal Lobe in Semantic Representation and Control: Priming Multiple versus Single Meanings of Ambiguous Words. Cereb Cortex. 21. 831-844. http://doi.org/10.1093/cercor/bhq148. 
Chao-Gan, Y., \& Yu-Feng, Z., 2010. DPARSF: A MATLAB Toolbox for "Pipeline" Data Analysis of RestingState fMRI. Front Syst Neurosci. 4. 13. http://doi.org/10.3389/fnsys.2010.00013.

Coutinho, J. F., Fernandesl, S. V., Soares, J. M., Maia, L., Goncalves, O. F., \& Sampaio, A., 2016. Default mode network dissociation in depressive and anxiety states. Brain Imaging Behav. 10. 147-157. http://doi.org/10.1007/s11682-015-9375-7.

Eguiluz, V. M., Chialvo, D. R., Cecchi, G. A., Baliki, M., \& Apkarian, A. V., 2005. Scale-free brain functional networks. Phys Rev Lett. 94. 018102. http://doi.org/10.1103/PhysRevLett.94.018102.

Eren, I., Tükel, R., Polat, A., Karaman, R., \& Unal, S., 2003. Evaluation of regional cerebral blood flow changes in panic disorder with Tc99m-HMPAO SPECT. Psychiatry Res. 123. 135-143. http://doi.org/10.1016/S0925-4927(03)00062-3.

Feldker, K., Heitmann, C. Y., Neumeister, P., Bruchmann, M., Vibrans, L., Zwitserlood, P., \& Straube, T., 2016. Brain responses to disorder-related visual threat in panic disorder. Hum Brain Mapp. 37. 4439-4453. http://doi.org/10.1002/hbm.23320.

Galderisi, S., Mancuso, F., Mucci, A., Garramone, S., Zamboli, R., \& Maj, M., 2008. Alexithymia and Cognitive Dysfunctions in Patients with Panic Disorder. Psychotherapy \& Psychosomatics. 77. 182-188. http://doi.org/10.1159/000119738

Goel, V., Gold, B., Kapur, S., \& Houle, S., 1998. Neuroanatomical Correlates of Human Reasoning. J Cogn Neurosci. 10. 293-302. http://doi.org/10.1162/089892998562744.

Gong, G., Rosa-Neto, P., Carbonell, F., Chen, Z. J., He, Y., \& Evans, A. C., 2009. Age- and gender-related differences in the cortical anatomical network. J Neurosci. 29. 15684-15693. http://doi.org/10.1523/JNEUROSCI.2308-09.2009.

Gorman, \& M., J., 2000. Neuroanatomical Hypothesis of Panic Disorder, Revised. Am J Psychiatry. 157. 493-505.

Han, D. H., Renshaw, P. F., Dager, S. R., Chung, A., Hwang, J., Daniels, M. A., . . Lyoo, I. K., 2008. Altered cingulate white matter connectivity in panic disorder patients. J Psychiatr Res. 42. 399-407. http://doi.org/10.1016/j.jpsychires.2007.03.002.

He, Y., \& Evans, A., 2010. Graph theoretical modeling of brain connectivity. Curr Opin Neurol. 23. 1. http://doi.org/10.1097/WC0.0b013e32833aa567.

Humphries, M. D., \& Gurney, K., 2008. Network 'small-world-ness': a quantitative method for determining canonical network equivalence. PLoS One. 3. e0002051. http://doi.org/10.1371/journal.pone.0002051.

Japee, S., Crocker, L., Carver, F., Pessoa, L., \& Ungerleider, L. G., 2009. Individual differences in valence modulation of face-selective M170 response. Emotion. 9. 59-69. http://doi.org/10.1037/a0014487. 
Kent, J. M., Coplan, J. D., Mawlawi, O., Martinez, J. M., Browne, S. T., Slifstein, M., . . Gorman, J. M., 2005. Prediction of Panic Response to a Respiratory Stimulant by Reduced Orbitofrontal Cerebral Blood Flow in Panic Disorder. American Journal of Psychiatry. 162. 1379-1381.

http://doi.org/10.1176/appi.ajp.162.7.1379.

Kessler, R. C., Chiu, W. T., Jin, R., Ruscio, A. M., Shear, K., \& Walters, E. E., 2006. The epidemiology of panic attacks, panic disorder, and agoraphobia in the National Comorbidity Survey Replication. Arch Gen Psychiatry. 63. 415-424. http://doi.org/10.1001/archpsyc.63.4.415.

Killgore, W. D. S., Britton, J. C., Schwab, Z. J., Price, L. M., Weiner, M. R., Gold, A. L., . . Rauch, S. L., 2014. CORTICO-LIMBIC RESPONSES TO MASKED AFFECTIVE FACES ACROSS PTSD, PANIC DISORDER, AND SPECIFIC PHOBIA. Depression \& Anxiety. 31. 150-159. http://doi.org/

10.1002/da.22156.

Lai, C.-H., Hsu, Y.-Y., \& Wu, Y.-T., 2010. First episode drug-naïve major depressive disorder with panic disorder: gray matter deficits in limbic and default network structures. Eur Neuropsychopharmacol. 20. 676-682. http://doi.org/10.1016/j.euroneuro.2010.06.002.

Lai, C.-H., \& Wu, Y.-T., 2014. The alterations in inter-hemispheric functional coordination of patients with panic disorder: the findings in the posterior sub-network of default mode network. J Affect Disord. 166. 279-284. http://doi.org/10.1016/j.jad.2014.05.022.

Lai, C. H., 2019. Fear Network Model in Panic Disorder: The Past and the Future. Psychiatry Investig. 16. 16-26. http://doi.org/10.30773/pi.2018.05.04.2.

Lai, C. H., \& Wu, Y. T., 2013. Changes in regional homogeneity of parieto-temporal regions in panic disorder patients who achieved remission with antidepressant treatment. J Affect Disord. 151. 709-714. http://doi.org/10.1016/j.jad.2013.08.006.

Lai, C. H., \& Wu, Y. T., 2016. The Explorative Analysis to Revise Fear Network Model for Panic Disorder: Functional Connectome Statistics. Medicine (Baltimore). 95. e3597.

http://doi.org/10.1097/MD.0000000000003597.

Latora, V., \& Marchiori, M., 2001. Efficient Behavior of Small-World Networks. Phys Rev Lett. 87. 198701. http://doi.org/10.1103/PhysRevLett.87.198701.

Lee, Y. S., Hwang, J., Kim, S. J., Sung, Y. H., Kim, J., Sim, M. E., . . Lyoo, I. K., 2006. Decreased blood flow of temporal regions of the brain in subjects with panic disorder. J Psychiatr Res. 40. 528-534. http://doi.org/10.1016/j.jpsychires.2005.08.012.

Leitman, D. I., Loughead, J., Wolf, D. H., Ruparel, K., Kohler, C. G., Elliott, M. A., . . Gur, R. C., 2008. Abnormal Superior Temporal Connectivity During Fear Perception in Schizophrenia. Schizophr Bull. 34. 673-678. http://doi.org/10.1093/schbul/sbn052.

Page $14 / 22$ 
Liao, W., Chen, H., Feng, Y., Mantini, D., Gentili, C., Pan, Z., ... Zhang, W., 2010. Selective aberrant functional connectivity of resting state networks in social anxiety disorder. Neuroimage. 52. 1549-1558. http://doi.org/10.1016/j.neuroimage.2010.05.010.

Liu, Y., Liang, M., Zhou, Y., He, Y., Hao, Y., Song, M., . . Jiang, T., 2008. Disrupted small-world networks in schizophrenia. Brain. 131. 945-961. http://doi.org/10.1093/brain/awn018.

Lueken, U., Straube, B., Reinhardt, I., Maslowski, N. I., \& Kircher, T., 2014. Altered top-down and bottom-up processing of fear conditioning in panic disorder with agoraphobia. Psychol Med. 44. 381-394. http://doi.org/10.1017/S0033291713000792.

Lynall, M. E., Bassett, D. S., Kerwin, R., McKenna, P. J., Kitzbichler, M., Muller, U., \& Bullmore, E., 2010. Functional Connectivity and Brain Networks in Schizophrenia. Journal of Neuroscience the Official Journal of the Society for Neuroscience. 30. 9477-9487. http://doi.org/10.1523/JNEUROSCI.033310.2010 .

Macaluso, E., Frith, C., \& Driver, J., 2000. Selective spatial attention in vision and touch: unimodal and multimodal mechanisms revealed by PET. J Neurophysiol. 83. 3062-3075.

http://doi.org/10.1152/jn.2000.83.5.3062.

Maddock, R. J., Buonocore, M. H., Kile, S. J., \& Garrett, A. S., 2003. Brain regions showing increased activation by threat-related words in panic disorder. Neuroreport. 14. 325-328.

http://doi.org/10.1097/00001756-200303030-00006.

Massana, G., Serra-Grabulosa, J. M., Salgado-Pineda, P., Gasto, C., Junque, C., Massana, J., \& Mercader, J. M., 2003. Parahippocampal gray matter density in panic disorder: a voxel-based morphometric study. Am J Psychiatry. 160. 566-568. http://doi.org/10.1176/appi.ajp.160.3.566.

Mcdermott, K. B., Petersen, S. E., Watson, J. M., \& Ojemann, J. G., 2003. A procedure for identifying regions preferentially activated by attention to semantic and phonological relations using functional magnetic resonance imaging. Neuropsychologia. 41. 293-303. http://doi.org/10.1016/S00283932(02)00162-8.

McIntosh, R. C., Hoshi, R. A., \& Timpano, K. R., 2020. Take my breath away: Neural activation at breathhold differentiates individuals with panic disorder from healthy controls. Respiratory physiology \& neurobiology. 277. 103427-103427. http://doi.org/10.1016/j.resp.2020.103427.

Mirz, F., Ovesen, T., Ishizu, K., Johannsen, P., Madsen, S., Gjedde, A., \& Pedersen, C. B., 1999. Stimulusdependent central processing of auditory stimuli: A PET study. Scandinavian Audiology. 28. 161-169. http://doi.org/10.1080/010503999424734.

Nash, J. R., Sargent, P. A., Rabiner, E. A., Hood, S. D., Argyropoulos, S. V., Potokar, J. P., . . Nutt, D. J., 2008. Serotonin 5-HT1A receptor binding in people with panic disorder: positron emission tomography study. 
British Journal of Psychiatry. 193. 229-234. http://doi.org/10.1192/bjp.bp.107.041186.

Qiu, C., Liao, W., Ding, J., Feng, Y., Zhu, C., Nie, X., . . Gong, Q., 2011. Regional homogeneity changes in social anxiety disorder: A resting-state fMRI study. Psychiatry Research: Neuroimaging. 194. 47-53. http://doi.org/10.1016/j.pscychresns.2011.01.010.

Quirk, G. J., Garcia, R., \& González-Lima, F., 2006. Prefrontal Mechanisms in Extinction of Conditioned Fear. Biol Psychiatry. 60. 337-343. http://doi.org/10.1016/j.biopsych.2006.03.010.

Reinecke, A., Filippini, N., Berna, C., Western, D. G., Hanson, B., Cooper, M. J., . . Harmer, C. J., 2015. Effective emotion regulation strategies improve fMRI and ECG markers of psychopathology in panic disorder: implications for psychological treatment action. Transl Psychiatry. 5. e673. http://doi.org/10.1038/tp.2015.160.

Sato, W., Toichi, M., Uono, S., \& Kochiyama, T., 2012. Impaired social brain network for processing dynamic facial expressions in autism spectrum disorders. BMC Neurosci. 13. 99-. http://doi.org/10.1186/1471-2202/13/99.

Sehlmeyer, C., Schöning, S., Zwitserlood, P., Pfleiderer, B., Kircher, T., Arolt, V., \& Konrad, C., 2009. Human Fear Conditioning and Extinction in Neuroimaging: A Systematic Review. PLoS One. 4. e5865. http://doi.org/10.1371/journal.pone.0005865.

Sobanski, T., \& Wagner, G., 2017. Functional neuroanatomy in panic disorder: Status quo of the research. World J Psychiatry. 7. 12-33. http://doi.org/10.5498/wjp.v7.i1.12.

Sobanski, T., Wagner, G., Peikert, G., Gruhn, U., Schluttig, K., Sauer, H., \& Schlosser, R., 2010. Temporal and right frontal lobe alterations in panic disorder: a quantitative volumetric and voxel-based morphometric MRI study. Psychol Med. 40. 1879-1886. http://doi.org/10.1017/S0033291709991930.

Sotres-Bayon, F., Cain, C. K., \& LeDoux, J. E., 2006. Brain mechanisms of fear extinction: historical perspectives on the contribution of prefrontal cortex. Biol Psychiatry. 60. 329-336. http://doi.org/10.1016/j.biopsych.2005.10.012.

Spoormaker, V. I., Andrade, K. C., Schr?ter, M. S., Sturm, A., Goya-Maldonado, R., S?mann, P. G., \& Czisch, M., 2011. The neural correlates of negative prediction error signaling in human fear conditioning. Neuroimage. 54. 2250-2256. http://doi.org/10.1016/j.neuroimage.2010.09.042.

Tauscher, J., Bagby, R. M., Javanmard, M., Christensen, B. K., \& Kapur, S., 2001. Inverse Relationship Between Serotonin 5-HT 1A Receptor Binding and Anxiety: A [ 11 C]WAY-100635 PET Investigation in Healthy Volunteers. American Journal of Psychiatry. 158. 1326-1328.

http://doi.org/10.1176/appi.ajp.158.8.1326.

Tzourio-Mazoyer, N., Landeau, B., Papathanassiou, D., Crivello, F., Etard, O., Delcroix, N., . . Joliot, M., 2002. Automated anatomical labeling of activations in SPM using a macroscopic anatomical 
parcellation of the MNI MRI single-subject brain. Neuroimage. 15. 273-289. http://doi.org/10.1006/nimg.2001.0978.

van de Riet, W. A. C., Grezes, J., \& de Gelder, B., 2009. Specific and common brain regions involved in the perception of faces and bodies and the representation of their emotional expressions. Social neuroscience. 4. 101-120. http://doi.org/10.1080/17470910701865367.

van den Heuvel, M. P., Stam, C. J., Boersma, M., \& Hulshoff Pol, H. E., 2008. Small-world and scale-free organization of voxel-based resting-state functional connectivity in the human brain. Neuroimage. 43. 528-539. http://doi.org/10.1016/j.neuroimage.2008.08.010.

Wang, \& Jinhui, 2010. Graph-based network analysis of resting-state functional MRI. Front Syst Neurosci. 4. 1-14. http://doi.org/10.3389/fnsys.2010.00016.

Wang, J., Wang, X., Xia, M., Liao, X., Evans, A., \& He, Y., 2015. GRETNA: A graph theoretical network analysis toolbox for imaging connectomics. Front Hum Neurosci. 9. 386.

http://doi.org/10.3389/fnhum.2015.00386.

Wang, L., Zhu, C., He, Y., Zang, Y., Cao, Q., Zhang, H., ... Wang, Y., 2009. Altered small-world brain functional networks in children with attention-deficit/hyperactivity disorder. Hum Brain Mapping. 30. 638649. http://doi.org/10.1002/hbm.20530.

Watts, D. J., \& Strogatz, S. H., 1998. Collective dynamics of 'small-world' networks. Nature. 393. 440. http://doi.org/10.1038/30918.

Wedekind, D., Gruber, O., Obst, K., Dechent, P., Engel, K., Ulrich, K., \& Bandelow, B., 2011. FC25-02 Functional MRI activation in response to panic-specific, non-panic aversive, and neutral imagery in patients with panic disorder and healthy controls. European Psychiatry - EUR PSYCHIAT. 26. 1954-1954. http://doi.org/10.1016/S0924-9338(11)73657-4.

Wig, G. S., Schlaggar, B. L., \& Petersen, S. E., 2011. Concepts and principles in the analysis of brain networks. Ann N Y Acad Sci. 1224. 126-146. http://doi.org/10.1111/j.1749-6632.2010.05947.x.

Wu, Y., Zhong, Y., Ma, Z., Lu, X., Zhang, N., Fox, P. T., \& Wang, C., 2018. Gray matter changes in panic disorder: A voxel-based meta-analysis and meta-analytic connectivity modeling. Psychiatry Research: Neuroimaging. 282. 82-89. http://doi.org/10.1016/j.pscychresns.2018.09.009.

Xia, M., Wang, J., \& He, Y., 2013. BrainNet Viewer: a network visualization tool for human brain connectomics. PLoS One. 8. e68910. http://doi.org/10.1371/journal.pone.0068910.

Yan, C. G., Wang, X. D., Zuo, X. N., \& Zang, Y. F., 2016. DPABI: Data Processing \& Analysis for (RestingState) Brain Imaging. Neuroinformatics. 14. 339-351. http://doi.org/10.1007/s12021-016-9229-4. 
Zalesky, A., Fornito, A., \& Bullmore, E. T., 2010. Network-based statistic: identifying differences in brain networks. Neuroimage. 53. 1197-1207. http://doi.org/10.1016/j.neuroimage.2010.06.041.

Zhang, J., Wang, J., Wu, Q., Kuang, W., Huang, X., He, Y., \& Gong, Q., 2011. Disrupted brain connectivity networks in drug-naive, first-episode major depressive disorder. Biol Psychiatry. 70. 334-342. http://doi.org/10.1016/j.biopsych.2011.05.018.

Zhu, H., Qiu, C., Meng, Y., Yuan, M., Zhang, Y., Ren, Z., . . Zhang, W., 2017. Altered Topological Properties of Brain Networks in Social Anxiety Disorder: A Resting-state Functional MRI Study. Sci Rep. 7. 43089. http://doi.org/10.1038/srep43089.

\section{Figures}




\section{A Altered Network}

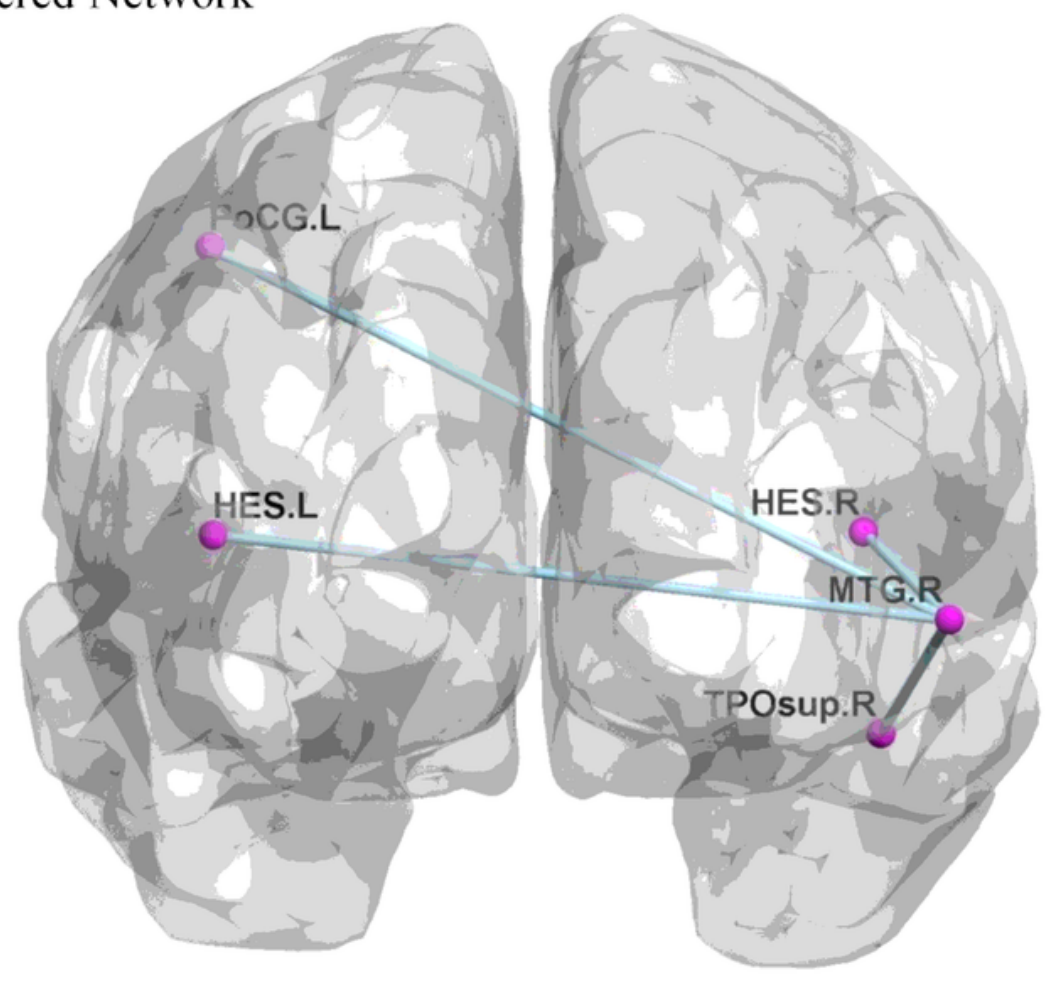

B Association between FC and symptoms

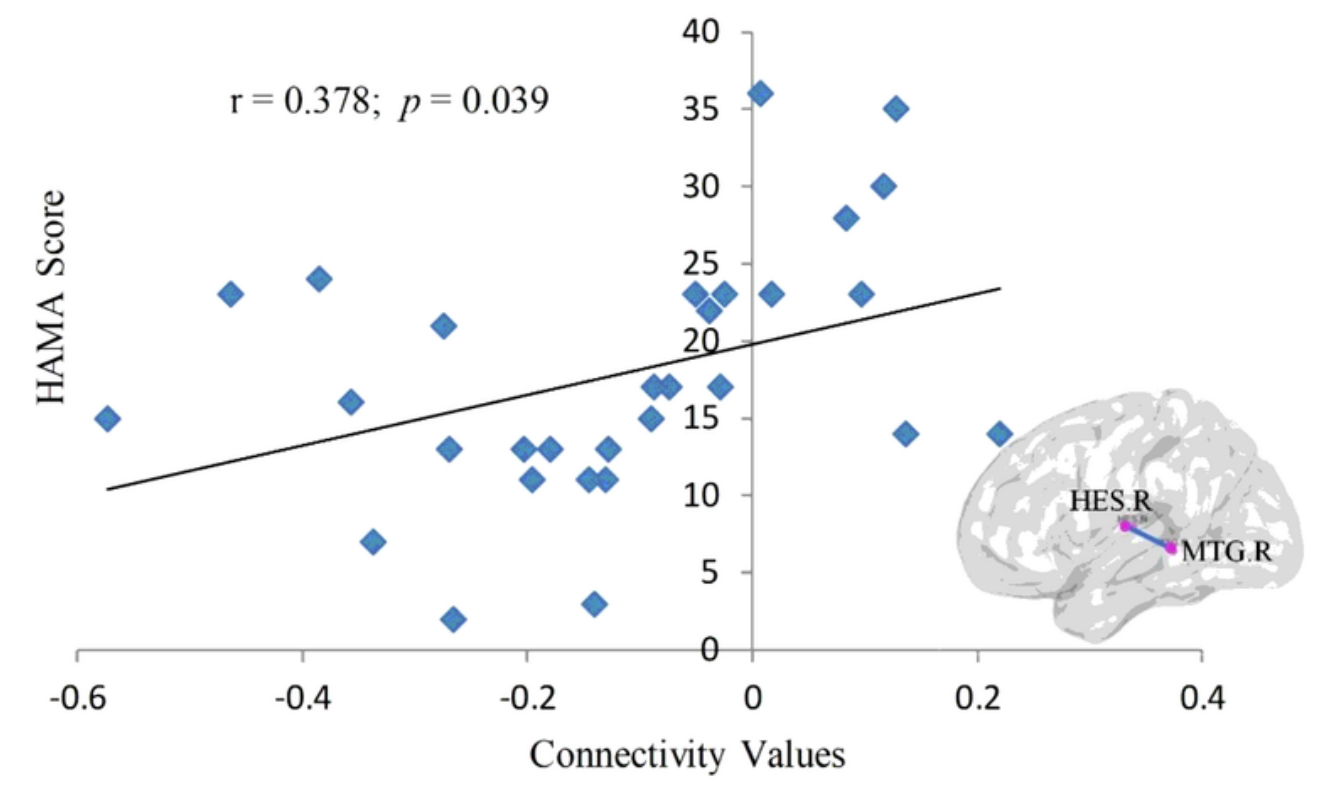

Figure 1

A, altered network in PD patients. PoCG.L, left postcentral gyrus; HES.L, left transverse temporal gyrus; HES.R, right transverse temporal gyrus; MTG.R, right middle temporal gyrus; TPOsup.R, right temporal pole: superior temporal gyrus. $\mathrm{B}$, correlation between $\mathrm{FC}$ values in the right $\mathrm{MTG}$ and right transverse temporal gyrus connection and HAMA scores. 
A Clustering coefficient

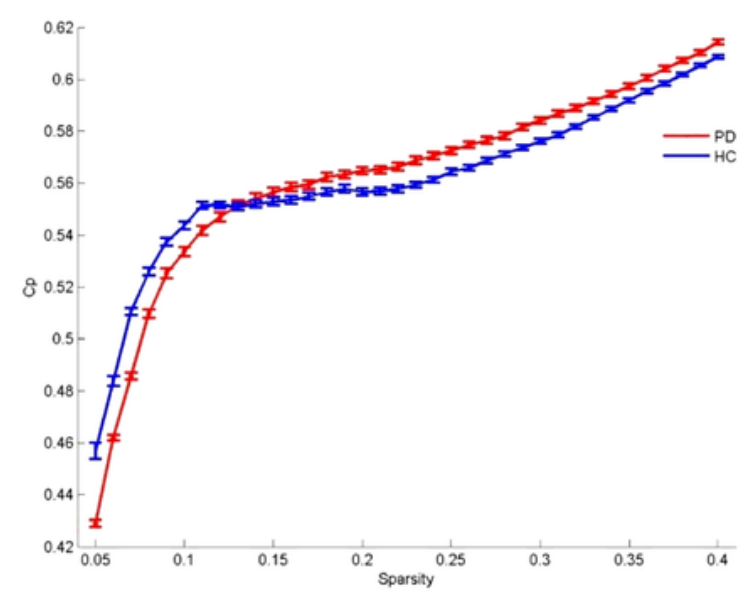

B Characteristic path length

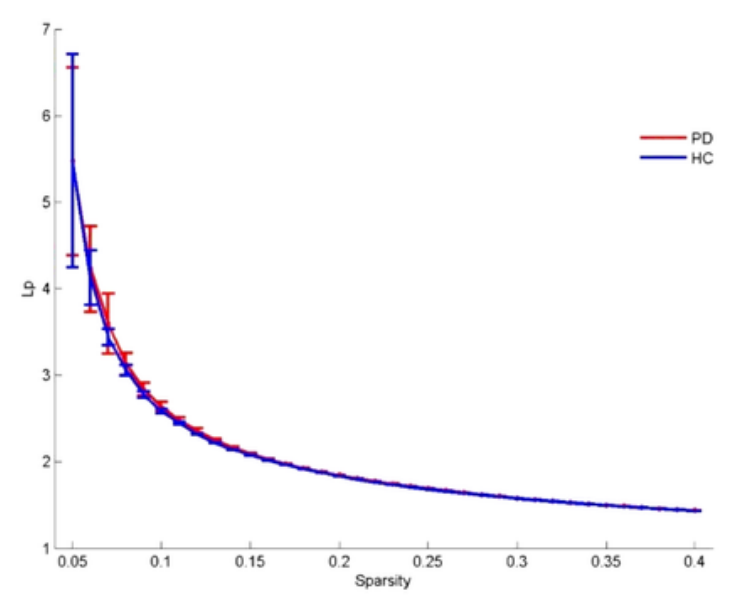

C Small-worldness

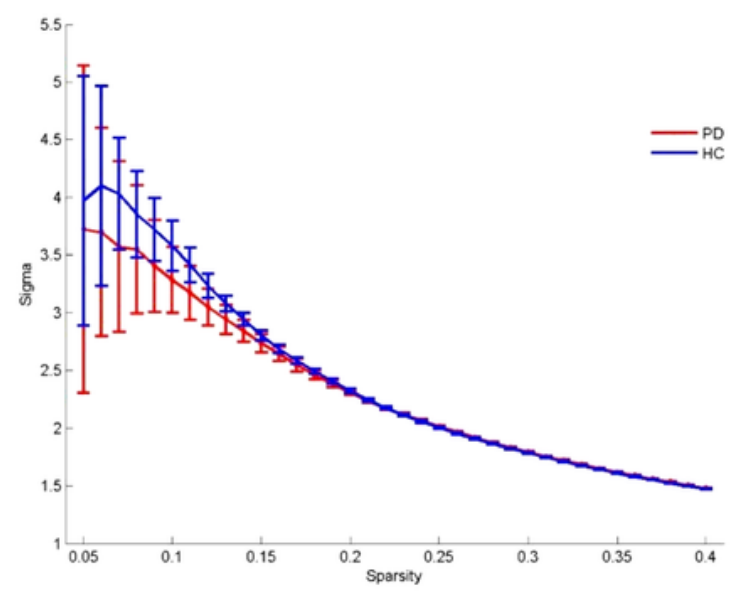

\section{Figure 2}

Measures of global topological properties of brain connectivity in PD and $\mathrm{HC}$ groups. A, Clustering coefficient (Cp); B, Characteristic path length (Lp); C, Small-worldness ( $\sigma)$. 


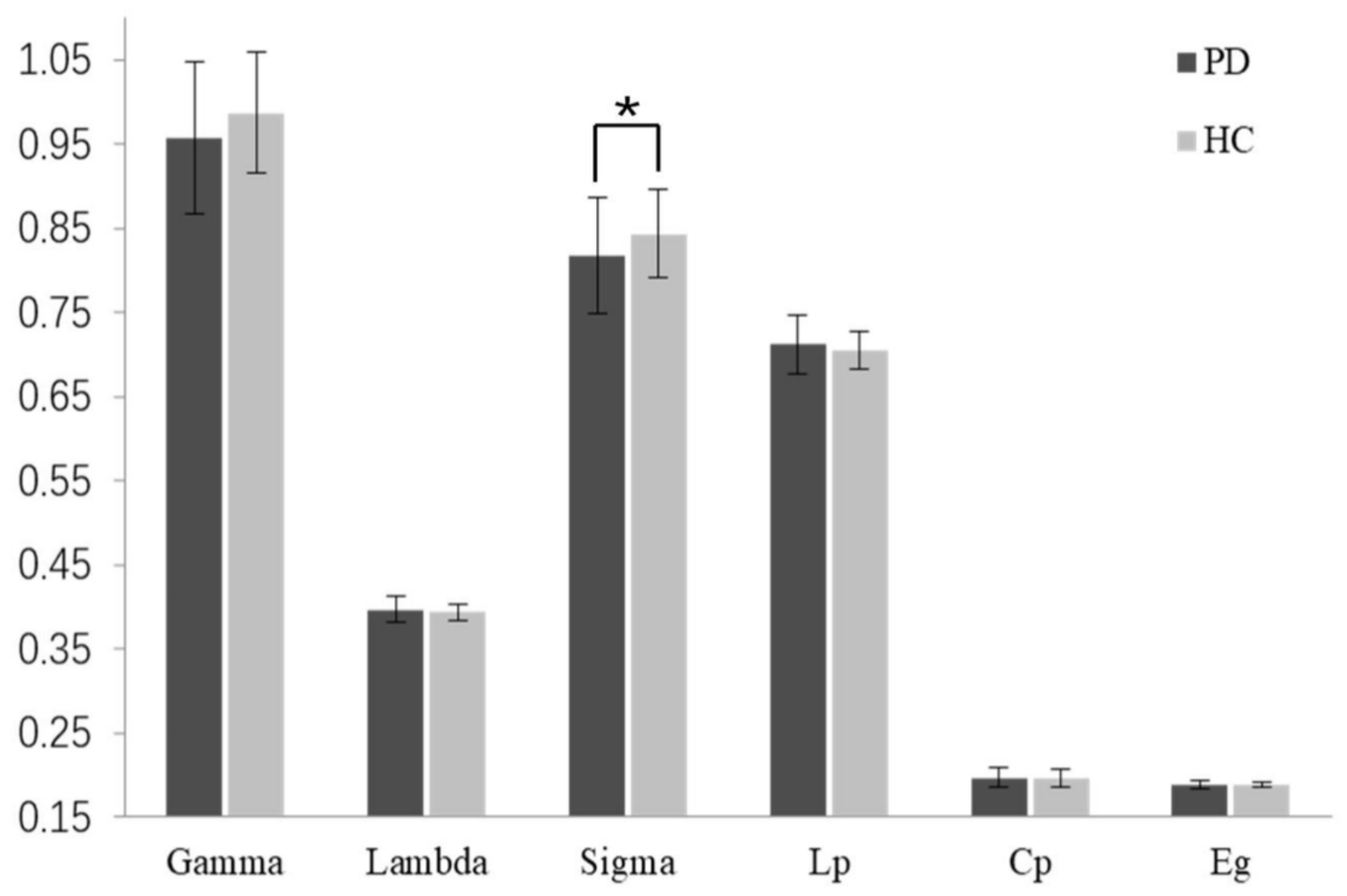

Figure 3

The AUC of global topological properties in PD and HC groups. * represents significant group difference at 0.05 level. Gamma $(\gamma)$, the normalized clustering coefficient; Lambda $(\lambda)$, the normalized characteristic path length; Sigma $(\sigma)$, small-world measures; Lp, shortest path length; $\mathrm{Cp}$, clustering coefficient; $\mathrm{Eg}$, global efficiency. 

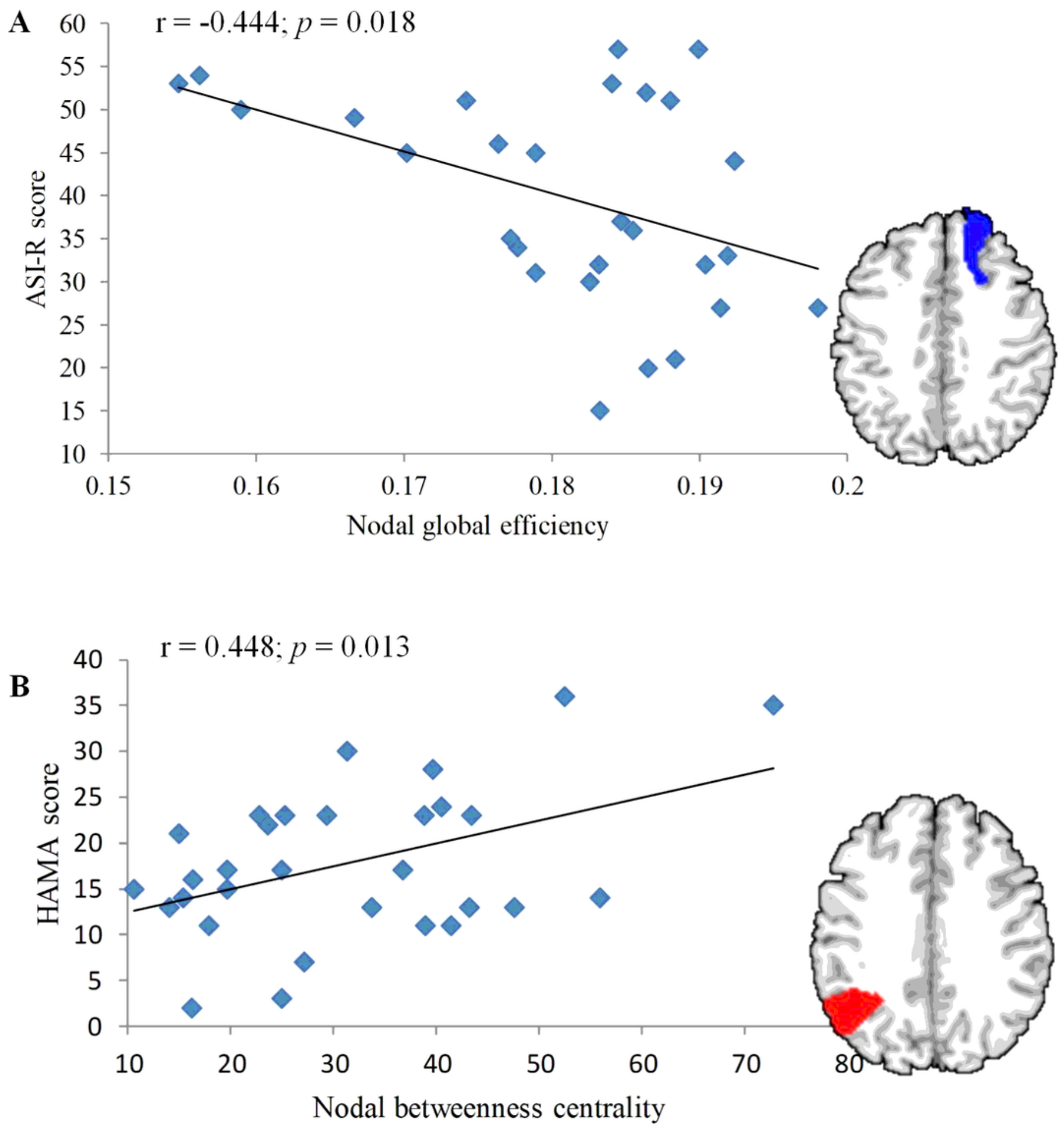

Figure 4

A, correlation between the AUC of nodal global efficiency of right superior frontal gyrus and ASI-R scores; $B$, correlation between the AUC of nodal betweenness centrality of left angular gyrus and HAMA scores. 